\title{
Predictors of mortality in blood stream infections caused by extended spectrum beta lactamase producing enterobacteriaceae
}

\author{
Chavada $\mathbf{R}^{1 *}$, Descallar $\mathrm{J}^{2,3}$ and Maley $\mathbf{M}^{4}$ \\ ${ }^{1}$ Department of Microbiology and Infectious Diseases, Pathology North, Gosford, NSW, Australia \\ ${ }^{2}$ Ingham Institute of Medical Research, Liverpool, NSW, Australia \\ ${ }^{3}$ South Western Sydney Clinical School, UNSW, Liverpool, NSW, Australia \\ ${ }^{4}$ Department of Microbiology and Infectious Diseases, Liverpool, Sydney South West Pathology Service, Liverpool, NSW, Australia
}

\begin{abstract}
Introduction: Extended spectrum beta-lactamases (ESBL) have been increasing worldwide and are associated with increased rates of treatment failure, morbidity, healthcare costs and mortality. This study was performed to evaluate the risk factors for mortality in ESBL blood stream infections.

Methods: A descriptive retrospective observational case study was performed. Data was collected from patient medical records and laboratory information system. Phenotypic resistance testing and multiplex PCR assay was done. Standard definitions were used. Standard bivariate analysis and multivariate analysis were performed.

Results: A total of 114 patients were included in this study. 30 day mortality rate was 18.4\% (21/114). Median APACHE2 score was higher in patients who died compared to those who survived [19.5 vs 16.4, $\mathrm{p}=0.002$ ]. Urinary tract infection $(61.7 \%)$ and intrabdominal (22.8 \%) were the most common source of infection. On univariate analysis patients who presented with intraabdominal infection (OR 3.3[1.1-10.3, $\mathrm{p}=0.03])$, received third generation cephalosporin (OR 23[2.27-233, $\mathrm{p}=0.008])$ as their definitive therapy, received inappropriate definitive (OR 28[5.3-146.5, $\mathrm{p}=<0.01$ ) and had a higher APACHE2 score $>20$ ( $\mathrm{p}=0.008)$ were associated with higher mortality at 30 days. Also patients who did not receive immunosuppressive medications $(\mathrm{OR} 0.26[0.08-0.86$, $\mathrm{p}=0.02])$ had lower mortality at 30 days compared to those did. On multivariate analysis receipt of inappropriate antimicrobial therapy (OR 26.21[4.75-144.93, p=0.001] and APACHE2 score > 20 (OR $3.48[1.12-10.74, \mathrm{p}=0.03]$ were independently associated with increased mortality.
\end{abstract}

Conclusions: Prompt institution of appropriate antimicrobial therapy (including a change from third generation cephalosporin) based on susceptibility results should be done in patients with ESBL BSI. Patients who receive inappropriate definitive antimicrobial therapy and those that present in septic shock have higher mortality with ESBL BSI.

\section{Introduction}

Extended spectrum betalactamase (ESBL) producing Enterobacteriaciae have become frequent worldwide, causing increased hospital morbidity and mortality $[1,2]$. The presence of this enzyme significantly reduces the available antimicrobial options, as ESBL genes are associated with other resistance encoding genes, which generates resistance to multiple classes of antibiotics $[3,4]$. The epidemiology of infections caused by these organisms has evolved and varies considerably according to geographic regions [5-7]. According to the European Antimicrobial surveillance network data, in 2012, the third generation cephalosporin resistance in Escherichia coli was $11.9 \%$ and in Klebsiella pneumoniae was $25.6 \%$, and this had increased over the preceding surveillance period [8].

There has been a great heterogeneity of the published studies that have looked at the risk factors for blood stream infections and mortality caused by ESBL producing Enterobacteriaceae [9]. While some have looked at particular organisms or groups of organisms, others have looked at the place of acquisition of infection such as hospital vs community onset. We undertook this study to understand the local epidemiology of ESBL producing enterobacteriaceae in south western Sydney and to determine outcomes of these infections in patients presenting to our hospitals. The aim of this study was to assess the risk factors for mortality for blood stream infections (BSI) caused by ESBL producing enterobacteriaceae in our area.

\section{Methods}

\section{Study setting and design}

South Western Sydney Local Health District covers a large urban and semi-rural area with a population of approximately 1 million people. The population is generally of lower socioeconomic status, has relatively poorer health and is very ethnically diverse. Many residents have close contact with overseas visitors and travel periodically to regional countries to visit friends and relatives. The Sydney South West

Correspondence to: Chavada R, Department of Microbiology and Infectious Diseases, Pathology North, Gosford, NSW, Australia, E-mail: ruchirchavda@ gmail.com

Key words: ESBL, bacteraemia, mortality, risk factors

Received: August 10, 2017; Accepted: September 19, 2017; Published: September 22, 2017 
Pathology Service (SSWPS) Microbiology laboratory provides services to all of the large tertiary care hospitals in the region comprising over 1600 acute hospital beds. Routine active surveillance for multi-drug resistant (MDR) gram negative (GN) organisms is performed on the 60 plus ICU beds and on selected high risk admissions.

A descriptive retrospective observational study was performed over a period of 3 years (January 2011- January 2014) where all cases of bacteraemia by ESBL producing enterobacteriaceae were included. The patients were identified from the microbiology laboratory information system and cross matched against stored organism database to ensure almost all ESBL BSI episodes were captured. The study was approved by the local South Western Sydney Human Research Ethics committee.

\section{Clinical data}

Electronic medical records were accessed to gather demographic and clinical information such as age, sex, underlying disease, primary site of infection, severity of illness as calculated by the Acute Physiology and Chronic Health Evaluation (APACHE) 2 score, duration of hospital stay, antimicrobial regimen, surgical procedure performed, overseas travel, presence of indwelling catheters, nursing home admissions and any antimicrobial therapy in the 90 days prior to onset of bacteraemia. Comorbidities recorded included neutropenia, presentation with septic shock, intensive care unit admission, immune suppressive agents within 60 days prior to onset of bacteraemia, corticosteroid and chemotherapy. The primary clinical outcome assessed was 30 day mortality.

\section{Definitions}

'Nosocomial infection' was defined as an infection that occurred $48 \mathrm{~h}$ after admission to the hospital, an infection that occurred $48 \mathrm{~h}$ after admission to the hospital in patients who had been hospitalized in the 2 weeks prior to admission, or an infection that occurred $48 \mathrm{~h}$ after admission to the hospital in patients that had been transferred from another hospital or nursing home. All other BSI was classified to be 'community onset'. Immunosuppression was defined as receipt of chemotherapy for haematological or solid organ malignancy or use of known immunosuppressive agents for treatment of other established conditions.

Multi-drug resistant (MDR) was defined as resistance to $\geq 3$ different classes of antimicrobial agents.

'Empirical therapy' was defined as antimicrobials given before the results of blood culture were available and 'definitive therapy' was defined as treatment given after the results of antibiotic susceptibility tests had been received. The antimicrobial therapy was considered 'appropriate' if the treatment regimen included antibiotics active in vitro with its dosage and route of administration in conformity with current medical standards. Cephalosporin monotherapy was considered inappropriate definitive therapy regardless of the minimal inhibitory concentration (MIC). Similarly when patients continued to received piperacillin-tazobactam or cefepime despite final results showing the organism being resistant was also considered as inappropriate. However the final decision about which antimicrobial to use was not up to the investigators but to the treating physicians, although all occasions of ESBL BSI were communicated to clinical teams by the microbiology laboratory and this included appropriate advice on use of antimicrobials.

\section{Microbiological methods}

Antimicrobial susceptibility testing and phenotypic confirmation of MDR GN was performed according to Clinical Laboratory
Standards Institute (CLSI) recommendations. Vitek2 (bioMerieux, Marcy l'Etoile, France) MIC profile was used as a screening test that lead to phenotypic, and sometimes genotypic, confirmatory tests. For enterobacteriaceae an MIC $\geq 2 \mathrm{mg} / \mathrm{L}$ for any one of the third generation cephalosporins (3GC), or cefepime (FEP), or ceftazidime (CAZ), or a gentamicin MIC $\geq 4 \mathrm{mg} / \mathrm{L}$, or a meropenem (MEM) MIC of $\geq 0.5 \mathrm{mg} / \mathrm{L}$, triggers further testing. The confirmatory phenotypic test was a combination of double disc synergy testing (DDST) and disc approximation (DA) used in tandem on a combination of Mueller Hinton agar (MHA) plates (CLSI). A 0.5 McFarland suspension was used to make a lawn and commercially produced antibiotic discs were placed at pre-set positions according to our locally validated method for detection of ESBL, AmpC and carbapenemases [10]. Also a locally validated commercial multiplex PCR was performed for detection of $\operatorname{ESBL}\left(\right.$ bla $_{\mathrm{TEM}}$, bla $\left._{\mathrm{SHV}}, \mathrm{bla}_{\mathrm{CTXM1}}, \mathrm{bla}_{\mathrm{CTXM9}}\right), \operatorname{AmpC}\left(\mathrm{bla}_{\mathrm{DHA}}, \mathrm{bla}_{\mathrm{ACC}}, \mathrm{bla}_{\mathrm{CMY}}\right)$ and carbapenemase $\left(\mathrm{bla}_{\mathrm{NDM}}, \mathrm{bla}_{\mathrm{KPC}}, \mathrm{bla}_{\mathrm{OXA23}}, \mathrm{bla}_{\mathrm{IMP}}\right)[11]$.

\section{Statistical analysis}

Student $t$ test was used to compare continuous variables, and Fisher exact test was used to compare categorical variables. Binomial univariate analysis was performed followed by multivariate backward logistic regression analysis using SAS version 9.1(Lane Cove, NSW, Australia).

\section{Results}

The median age of the patients in the study was 71.1 years with $61 \%(70 / 114)$ patients being male. Community associated ESBL BSI was more frequently seen (64\%). Comorbidities were seen in both cases and controls including haematological and solid organ malignancy, diabetes, chronic renal impairment (with or without dialysis), chronic liver disease from alcohol or chronic hepatitis infection. On univariate analysis, there was no statistical difference found in age, sex, onset or comorbidities in the patients who died at 30 days. 30 day mortality related to ESBL BSI was $18.4 \%(21 / 114)$ (Table 1$)$.

We found that 25/114(21.9\%) were from a nursing home, $10 / 114(8.7 \%)$ had travelled overseas in the last 6 months and $23 / 114(20.1 \%)$ had recent surgery in the last 3 months. Similarly on univariate analysis these factors did not differ between the two patient subsets. Similarly other features that were studied included previous hospital admission $(76 / 114,66.6 \%)$ for any reason (in the last 3 months), admission to intensive care unit $(27 / 114,23.6 \%)$, antibiotics administered in the past 60 days $(40 / 114,35 \%)$ and presence of urinary catheter for more than 6 weeks $(19 / 114,16.6 \%)$. There was no difference found in terms of mortality in these variables between the subsets. Immunosuppression was more frequent in the subset of patients who had died as opposed to those who survived ( 28.5 vs. 7.8, $\mathrm{p}=0.02)$.

Urinary tract infections (60.5\%) were most commonly seen in this study followed by intraabdominal (22.8\%), pneumonia (7\%), skin soft tissue infections (3.5\%), central line associated (3.5\%), and bone and joint infections (2.6\%). Escherichia coli was the most common organism $(79.8 \%)$, followed by Klebsiella pneumoniae (14\%) and Enterobacter cloacae (4\%).

CTX-M1 was the most type of ESBL genotype (47.6\%) followed CTX-M9 (33.3\%) (Figure 1).

Inappropriate empirical antimicrobial therapy was given in $16 / 21(76 \%)$ of patients who died, as opposed to $81 / 93(87 \%)$ of patients who had survived. There was no statistical difference found between the two groups. Inappropriate definitive therapy constituted cephalosporin 
Table 1. Clinical characteristics of patients with ESBL BSI

\begin{tabular}{|c|c|c|c|c|c|c|c|c|}
\hline \multirow[t]{2}{*}{ Parameter } & \multicolumn{2}{|c|}{$\operatorname{Died}(\mathrm{n}=21)$} & \multicolumn{2}{|c|}{ Survived(n=93) } & \multicolumn{4}{|c|}{$95 \%$ CI } \\
\hline & $\mathrm{n}$ & $\%$ & $\mathrm{n}$ & $\%$ & OR & Lower & Upper & P-value \\
\hline Age(years) & 76 & & 74 & & & & & 0.38 \\
\hline Sex: Male & 13 & 61.9 & 57 & 61.2 & 0.97 & 0.36 & 2.58 & 0.95 \\
\hline \multicolumn{9}{|l|}{ Onset } \\
\hline 1) Community & 12 & 57.1 & 61 & 65.5 & 0.69 & 0.26 & 1.83 & 0.46 \\
\hline 2) Hospital & 9 & 42.9 & 32 & 34.5 & & & & \\
\hline \multicolumn{9}{|l|}{ Organisms } \\
\hline Escherichia Coli & 16 & 76.1 & 75 & 80.6 & & & & \\
\hline Klebsiella pneumoniae & 3 & 14.2 & 13 & 13.9 & 1.08 & 0.21 & 4.24 & 0.91 \\
\hline Others & 2 & 9.5 & 5 & 5.3 & 1.17 & 0.12 & 11.19 & 0.89 \\
\hline \multicolumn{9}{|l|}{ Clinical diagnosis } \\
\hline 1) Urinary tract infection & 8 & 38 & 61 & 65.5 & & & & \\
\hline 2) Intraabdominal & 8 & 38 & 18 & 19.3 & 3.39 & 1.13 & 10.32 & 0.03 \\
\hline 3) Pneumonia & 6 & 35.2 & 11 & 64.7 & 1.69 & 0.31 & 9.23 & 0.54 \\
\hline \multicolumn{9}{|l|}{ Antibiotics Given } \\
\hline 1) Ciprofloxacin & 0 & 0 & 23 & 100 & & & & \\
\hline 2) Meropenem & 8 & 38 & 46 & 49.4 & 0.95 & 0.11 & 9.22 & 0.97 \\
\hline 3) Ceftriaxone & 4 & 19 & 1 & 1.0 & 23 & 2.27 & 233.19 & 0.008 \\
\hline 4) Piperacillin-tazobactam & 7 & 33.3 & 16 & 17.2 & 2.52 & 0.79 & 8.04 & 0.12 \\
\hline 5) Other-cefepime, gentamicin & 2 & 9.5 & 7 & 7.5 & 1.64 & 0.29 & 9.38 & 0.57 \\
\hline \multicolumn{9}{|l|}{ Comorbidities } \\
\hline 1) Malignancy & 8 & 38 & 19 & 20.4 & 0.41 & 0.15 & 1.15 & 0.09 \\
\hline 2) Diabetes & 3 & 14.2 & 19 & 20.4 & 1.54 & 0.41 & 5.77 & 0.52 \\
\hline 3) Renal failure & 4 & 19.0 & 10 & 10.7 & 0.51 & 0.14 & 1.82 & 0.30 \\
\hline 4) Liver disease & 3 & 14.2 & 5 & 5.3 & 0.34 & 0.07 & 1.55 & 0.16 \\
\hline History of Travel & 1 & 4.7 & 9 & 10.7 & 2.143 & 0.257 & 17.901 & 0.48 \\
\hline Surgery in hospital & 3 & 14.2 & 20 & 21.5 & 1.64 & 0.44 & 6.14 & 0.46 \\
\hline ICU admission & 3 & 14.2 & 24 & 25.8 & 2.08 & 0.56 & 7.71 & 0.27 \\
\hline Nursing home & 6 & 28.4 & 19 & 20.4 & 0.64 & 0.22 & 1.87 & 0.41 \\
\hline Length of hospital stay & 8 & & 11 & & & & & 0.29 \\
\hline APACHE2(median) score & 19.5 & & 16.4 & & & & & 0.002 \\
\hline APACHE2 $>20$ & 11 & 52.3 & 21 & 22.5 & & & & 0.008 \\
\hline Urologic procedure & 2 & 9.5 & 16 & 17.2 & 1.97 & 0.41 & 9.33 & 0.39 \\
\hline Previous hospitalisation(<3 months) & 17 & 80.9 & 59 & 63.4 & 0.40 & 0.12 & 1.31 & 0.13 \\
\hline Indwelling catheter & 3 & 14.2 & 16 & 17.2 & 1.24 & 0.32 & 4.74 & 0.74 \\
\hline Immunosuppressive medication & 6 & 28.4 & 9 & 9.6 & 0.26 & 0.08 & 0.86 & 0.02 \\
\hline Inappropriate empirical treatment & 5 & 23.8 & 12 & 12.9 & 0.47 & 0.14 & 1.53 & 0.21 \\
\hline Inappropriate definitive therapy & 8 & 38 & 2 & 2.1 & 28 & 5.3 & 146.5 & 0.01 \\
\hline
\end{tabular}

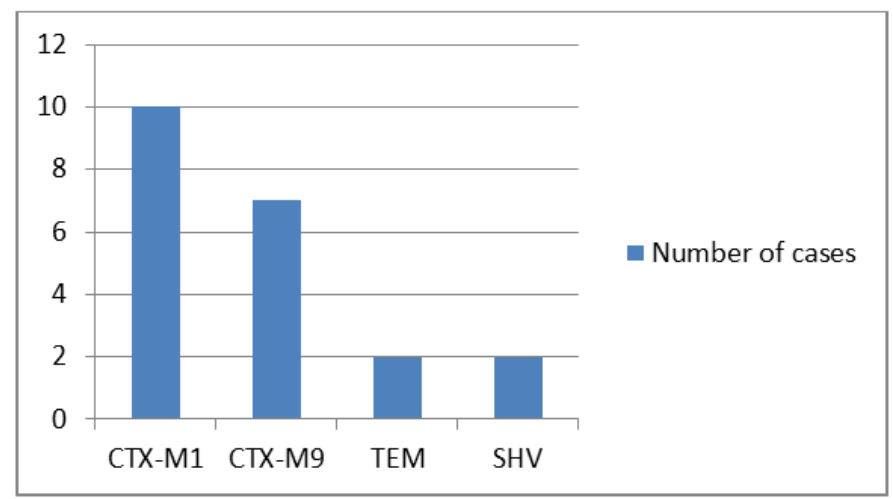

Figure 1. Predominant genotypes of ESBLs in patients who died.

monotherapy or combination of piperacillin -tazobactam or cefepime (when tested resistant by laboratory) given to patients despite results of the organism harbouring an ESBL enzyme being communicated to the treating clinician. This was given $8 / 21(38 \%)$ of patients who had died.

Median APACHE 2 score was higher in patients who had died as opposed to those who had survived (19.5 vs. $16.4, \mathrm{p}=0.002$ ). Median hospital length of stay was 17.2 days (IQR 1-166). Antimicrobials used in order of frequency were meropenem $(47.3 \%)$, ciprofloxacin $(20 \%)$, piperacillin-tazobactam $(20 \%)$, cefepime $(6.1 \%)$, ceftriaxone $(4.3 \%)$ and gentamicin $(1.7 \%)$.

Source control surgery or intervention appropriate for controlling source of sepsis was performed only in $23 \%$ of cases.

On univariate analysis patients who presented with intraabdominal infection ( $\mathrm{OR}$ 3.3[1.1-10.3, $\mathrm{p}=0.03])$, received third generation cephalosporin (OR 23[2.27-233, $\mathrm{p}=0.008]$ ) as their definitive therapy, received inappropriate definitive (OR 28[5.3-146.5, $\mathrm{p}=0.001)$ and had a higher APACHE2 score $>20(\mathrm{p}=0.008)$ were associated with higher mortality at 30 days. Also patients who did not receive immunosuppressive medications (OR 0.26[0.08-0.86, p=0.02]) had lower mortality at 30 days compared to those did. On multivariate analysis receipt of inappropriate antimicrobial therapy (OR 26.21 [4.75144.93, $\mathrm{p}=0.001]$ and APACHE2 score $>20($ OR 3.48[1.12-10.74, $\mathrm{p}=0.03$ ] were independently associated with increased mortality (Table 2).

\section{Discussion}

The incidence rates of ESBL in Australia has been looked at in community based Gram negative surveillance programs where 
Table 2. Multivariate analysis.

\begin{tabular}{|c|c|c|c|c|c|}
\hline \multicolumn{2}{|c|}{ Parameter } & \multirow{2}{*}{\begin{tabular}{|l|} 
OR \\
26.61
\end{tabular}} & \multicolumn{2}{|c|}{$95 \% \mathrm{CI}$} & \multirow{2}{*}{\begin{tabular}{|l|} 
P value \\
0.001
\end{tabular}} \\
\hline 1) & Inappropriate definitive therapy & & 4.75 & 144.93 & \\
\hline 2) & APACHE2 score $>\mathbf{2 0}$ & 3.48 & 1.12 & 10.74 & 0.03 \\
\hline
\end{tabular}

cumulative rates of $7.1 \%$ were found for Escherichia coli and $4.3 \%$ for Klebsiella pneumoniae. These rates are significantly lower than ESBL incidence rates from countries like China, India and southern European countries $(30-60 \%)[12,13]$. We found a crude mortality rate of $18.4 \%$ which falls in the range of mortality reported from other studies which range from $8.1-43.6 \%$. $[4,14,15]$ In our study we found that majority of ESBL BSI were community onset (64\%), which is similar to study by van Aken et al. [16] (68\%) in a low prevalence setting. However other studies have found a higher percentage of nosocomial onset (80\%) [17]. This difference could be related to the prevalence of ESBL in community setting and as well nosocomial factors like antimicrobial prescribing including antimicrobial stewardship, infection control practices in particular hospitals. Several other studies found that presence of more than 2 comorbidities increased the risk of mortality from ESBL BSI which was not observed in our study $[17,18]$. This difference could be attributed to the onset of the BSI as hospitalised patients with comorbidities in these studies as this group of patients are more likely to get such infections. Majority of ESBL BSI in our study was community onset infections. We did not find admission from Nursing home as a risk factor for mortality which was found in the study by Marachaim et al. [19].

While nosocomial onset of bacteraemia was found to be a risk factor for death in other studies we found that causes of such bacteraemias like surgery in hospital, presence of indwelling catheter, urological procedure and previous hospital admission in the 6 months were not associated with increased mortality [20]. We postulate that the difference in the epidemiology of infections and community onset of bacteraemias would be likely to contribute to this difference in our patient population. Like other studies we too found that patients with higher illness severity scores (APACHE2) had increased mortality [21,22]. Also a higher Pitt score and Charlson score have been independently associated with increased mortality in other studies $[14,23]$.

Patients on immunosuppressants' for haematological, solid organ malignancies and organ transplants had higher mortality in our study as was found the study by Park et al. [21] and Menashe et al. [24]. This is likely to result from presentation with neutropenia with sepsis/ septic shock requiring intensive care admission as was found in our study. Higher mortality was observed in our study in patients who had intraabdominal source of infection including biliary tract infections.

While inappropriate empirical therapy was found to be associated with increased mortality in several studies, we did not find this association. An important reason for this difference in finding could be attributed to the recommendation of Australian Therapeutic Guideline: Antibiotic (version 15, eTG) which suggests using intravenous gentamycin for 2 doses for all patients with suspected sepsis of unknown cause and in patients who present with overt sepsis to emergency department [25]. We found that $42 \%$ of isolates of ESBL in our study (data not shown) remained gentamycin susceptible which could explain this difference of outcome. However inappropriate definitive therapy was associated with increased mortality as was found in the study by Wang et al. [26]. One of the important reason for this finding in our study was due to continued use of third generation cephalosporin (Ceftriaxone) and betalactam-betalactamase inhibitor (Piperacillintazobactam) for definitive therapy for ESBL BSI. Continuation of these antimicrobials was the clinician's decision despite being notified of the ESBL status of the organism. We think that it may be due to fact that these patients presented with terminal medical conditions and were entering a palliative phase of care. However it is difficult to determine on this retrospective study if these patients deteriorated within first 4872 hours of admission due to sepsis (alone) and thus treating clinicians decided on palliative management. We did not find any differences in outcomes in patients who were treated with a carbapenem, fourth generation cephalosporin (cefepime) and piperacillin-tazobactam. On multivariate analysis higher APACHE2 score $>20$ and receipt of inappropriate antimicrobial therapy was independently associated with increased mortality in patients with ESBL BSI. Tumbarello et al. [27] also found that despite adequate treatment in patients with ESBL BSI, the mortality rate remained reasonably high (18.5\%) [27]. Higher APACHE2 score is also reflective that patients in our study who died presented with systemic inflammatory response syndrome (SIRS) and and/or severe sepsis. Leistner et al. [28] did not find that production of ESBL by $E$. Coli isolates was associated with increased mortality in their study. Similarly another study by Wu et al. [29] did not find any difference in mortality between different strains of CTX-m producing E Coli. We did not find that production of CTX-M genotype was associated with increased mortality in our study.

Harris et al. [30] found that there was no difference in outcomes of patients treated with beta lactam-betalactamase inhibitor combination (where susceptibility was proven and ESBL organism's MIC was within the sensitive breakpoint) comparted to a carbapenem. The MIC for piperacillin-tazobactam was $\leq 8 / 32$ for all isolates which tested "susceptible" by CLSI breakpoints. Interestingly there were no deaths observed in all patients who received a fluoroquinolone (ciprofloxacin) antibiotic as definitive therapy for ESBL BSI. Ciprofloxacin MIC by Vitek2 (BioMerieux, Marcy E'toile, France) was $\leq 0.25 \mu \mathrm{g} / \mathrm{ml}$ in all ESBL producing isolates who were treated with this antibiotic. While the issue of use of fluoroquinolone even when tested 'susceptible' has been debated due to reported failure in other studies, it is unclear as to whether the failure was due to the class of antibiotic itself [31-33]. Patients in these studies when treated with fluroquinolones had a higher MIC $0.38 \mu \mathrm{g} / \mathrm{ml}$, which still falls within the susceptible range but adequate tissue concentrations would not be achieved for treating high inoculum infections. Hence in carefully selected cases (MIC of $\leq 0.25$ $\mu \mathrm{g} / \mathrm{ml}$ ) of ESBL BSI, ciprofloxacin could be used as a carabapenem sparing agent or sequentially as a de-escalation antimicrobial.

However as with fluroquinolones there is a concern of treating infections with ESBL enterobacteriaceae with cefepime MIC of $>4$ due to risk of clinical failures [34]. All patients treated with cefepime in our study had a cefepime MIC of $\leq 2 \mu \mathrm{g} / \mathrm{ml}$ which could explain the favourable outcomes.

We acknowledge the limitations of our study. Being a retrospective study, outcomes especially related to choice of definitive antimicrobials could not be commented upon. With stronger presence of antimicrobial stewardship in our hospitals, there is more leverage for infectious diseases physicians to discuss and rationalise antimicrobial therapy particularly for treatment of multi drug resistance organisms. We only looked at the 30 day mortality in this study and it is not possible to assess the long term outcomes of patients who had ESBL BSI. Also strategies that have shown to reduce transmission of multi drug resistance organisms like routine active surveillance in 'high risk' units like ICU, haematology was possible in patients who were admitted to these wards. The burden of community ESBL carriage could not be assessed as routine screening in not performed in our hospital setting. 
Despite the above issues, this study identifies the risk factors associated with increased mortality in ESBL BSI in Australian setting which has not been previously studied.

\section{Conclusion}

Prompt institution of appropriate antimicrobial therapy (including a change from third generation cephalosporin) based on susceptibility results should be done in patients with ESBL BSI. Patients who receive inappropriate definitive antimicrobial therapy and those that present in septic shock have higher mortality with ESBL BSI.

Fluroquinolones could be considered in carefully selected cases (MIC of organism $\leq 0.25$ ) of ESBL BSI as a carbapenem sparing strategy or at de-escalation as oral therapy.

\section{References}

1. Rodríguez-Baño J, Pascual A (2008) Clinical significance of extended-spectrum betalactamases. Expert Rev Anti Infect Ther 6: 671-683. [Crossref]

2. Giske CG, Monnet DL, Cars O, Carmeli Y (2008) ReAct-Action on Antibiotic R. Clinical and economic impact of common multidrug-resistant gram-negative bacilli. Antimicrob Agents Chemother 52: 813-821.

3. Paterson DL, Bonomo RA (2005) Extended-spectrum beta-lactamases: a clinical update. Clin Microbiol Rev 18: 657-686. [Crossref]

4. Schwaber MJ, Carmeli Y (2007) Mortality and delay in effective therapy associated with extended-spectrum beta-lactamase production in Enterobacteriaceae bacteraemia: a systematic review and meta-analysis. J Antimicrob Chemother 60: 913-920.

5. Chong Y, Ito Y, Kamimura T (2011) Genetic evolution and clinical impact in extendedspectrum beta-lactamase-producing Escherichia coli and Klebsiella pneumoniae. Infection, genetics and evolution. J Molec Epidemiol Evol Gen Infect Dis 11: 1499-1504.

6. Cantón R, Novais A, Valverde A, Machado E, Peixe L, et al. (2008) Prevalence and spread of extended-spectrum beta-lactamase-producing Enterobacteriaceae in Europe. Clin Microbiol Infect 14 Suppl 1: 144-153. [Crossref]

7. Sidjabat HE, Paterson DL (2015) Multidrug-resistant Escherichia coli in Asia: epidemiology and management. Expert Rev Anti Infect Ther 13: 575-591. [Crossref]

8. ECDC SURVEILLANCE REPORT (2014) Annual epidemiological report, Antimicrobial resistance and healthcare-associated infections.

9. Trecarichi EM, Cauda R, Tumbarello M (2012) Detecting risk and predicting patient mortality in patients with extended-spectrum beta-lactamase-producing Enterobacteriaceae bloodstream infections. Future Microbiol 7: 1173-1189.

10. Chater M, Ziochos $\mathrm{H}$, Neville $\mathrm{S}$ (2011) Validation of a phenotypic screening method for ESBL, AmpC and mbl resistant mechanisms in clinical isolates of enterobacteriaceae, Proceedings of Australian Society for Antimicrobials 12th Annual Scientific Meeting (Antimicrobials) Melbourne 2011.

11. Chavada R, Maley M (2015) Evaluation of a Commercial Multiplex PCR for Rapid Detection of Multi Drug Resistant Gram Negative Infections. The Open Microbiology Journal 9: 125-135.

12. Australian Group on Antimicrobial Resistance Community-onset Gram-negative Surveillance Program annual report, 2010.

13. Pitout JD (2010) Infections with extended-spectrum beta-lactamase-producing enterobacteriaceae: changing epidemiology and drug treatment choices. Drugs 70 : 313-333. [Crossref]

14. Lee CC, Lee NY, Yan JJ, Lee HC, Chen PL, et al. (2010) Bacteremia due to extendedspectrum-beta-lactamase-producing Enterobacter cloacae: role of carbapenem therapy. Antimicrob Agents Chemother 54: 3551-3556.

15. Alvarez R, Vinas-Castillo L, Lepe-Jimenez JA, Garcia-Cabrera E, Cisneros-Herreros JM (2012) Time to positivity of blood culture association with clinical presentation, prognosis and ESBL-production in Escherichia coli bacteremia. Euro J Clin Microbiol Infect Dis 31: 2191-2195.

16. Van Aken S, Lund N, Ahl J, Odenholt I, Tham J (2014) Risk factors, outcome and impact of empirical antimicrobial treatment in extended-spectrum B-lactamaseproducing Escherichia coli bacteraemia. Scan J Infect Dis 46: 753-762.

17. Denis B, Lafaurie M, Donay JL, Fontaine JP, Oksenhendler E, et al. (2015) Prevalence, risk factors, and impact on clinical outcome of extended-spectrum beta-lactamaseproducing Escherichia coli bacteraemia: a five-year study. Inter J Infect Dis 39: 1-6.
18. Ortega M, Marco F, Soriano A, Almela M, Martinez JA, et al. (2009) Analysis of 4758 Escherichia coli bacteraemia episodes: predictive factors for isolation of an antibioticresistant strain and their impact on the outcome. J Antimicrob Chemother 63: 568-574.

19. Marchaim D, Gottesman T, Schwartz O, Korem M, Maor Y, et al. (2010) National multicenter study of predictors and outcomes of bacteremia upon hospital admission caused by Enterobacteriaceae producing extended-spectrum beta-lactamases Antimicrob Agents Chemother 54: 5099-5104.

20. Marra AR, Wey SB, Castelo A, Gales AC, Cal RG, et al. (2006) Nosocomia bloodstream infections caused by Klebsiella pneumoniae: impact of extended-spectrum beta-lactamase (ESBL) production on clinical outcome in a hospital with high ESBL prevalence. BMC Infect Dis 6: 24.

21. Park SH, Choi SM, Lee DG, Kim J, Choi JH, et al. (2011) Emergence of extendedspectrum beta-lactamase-producing escherichia coli as a cause of community-onset bacteremia in South Korea: risk factors and clinical outcomes. Microb drug Resis 17: 537-544.

22. Huang SS, Lee MH, Leu HS (2006) Bacteremia due to extended-spectrum betalactamase-producing Enterobacteriaceae other than Escherichia coli and Klebsiella. $J$ Microbiol Immun Infect 39: 496-502.

23. Kang CI, Chung DR, Ko KS, Peck KR, Song JH (2011) Korean Network for Study of Infectious D. Risk factors for infection and treatment outcome of extended-spectrum beta-lactamase-producing Escherichia coli and Klebsiella pneumoniae bacteraemia in patients with hematologic malignancy. Ann Hematol 91: 115-121.

24. Menashe G, Borer A, Yagupsky P, Peled N, Gilad J, et al. (2001) Clinical significance and impact on mortality of extended-spectrum beta lactamase-producing Enterobacteriaceae isolates in nosocomial bacteremia. Scand J Infect Dis 33: 188-193. [Crossref]

25. Australian Therapeutic guidelines, Antibiotics (version 15), Accessed from: https:// www.clinicalguidelines.gov.au/register/therapeutic-guidelines-antibiotic-version-15.

26. Wang SS, Lee NY, Hsueh PR, Huang WH, Tsui KC, et al. (2011) Clinical manifestations and prognostic factors in cancer patients with bacteremia due to extended-spectrum $\hat{\mathrm{I}}^{2}$ lactamase-producing Escherichia coli or Klebsiella pneumoniae. J Microbiol Immunol Infect 44: 282-288. [Crossref]

27. Tumbarello M, Sanguinetti M, Montuori E, Trecarichi E, Posteraro B, et al. (2007) Predictors of Mortality in Patients with Bloodstream Infections Caused by ExtendedSpectrum- $\beta$-Lactamase-Producing Enterobacteriaceae: Importance of Inadequate Initial Antimicrobial Treatment. Antimicrob Agents Chemother 51: 1987-1994.

28. Leistner R, Sakellariou C, Gurntke S, Kola A, Steinmetz I, et al. (2014) Mortality and molecular epidemiology associated with extended-spectrum beta-lactamase production in Escherichia coli from bloodstream infection. Infect Drug Resis 7: 57-62.

29. Wu UI, Wang JL, Chen WC, Chang SC, Chen YC (2011) Risk factors and outcomes of Escherichia coli bacteremia caused by strains that produce CTX-M or non-CTX-M extended-spectrum-beta-lactamases. Eur J Clin Microbiol Infect Dis 30: 33-39. [Crossref]

30. Harris PN, Yin M, Jureen R, Chew J, Ali J, et al. (2015) Comparable outcomes for $\hat{\mathrm{I}}^{2}$ lactam $/ \hat{I}^{2}$-lactamase inhibitor combinations and carbapenems in definitive treatment of bloodstream infections caused by cefotaxime-resistant Escherichia coli or Klebsiella pneumoniae. Antimicrob Resist Infect Control 4: 14. [Crossref]

31. Kim BN, Woo JH, Kim MN, Ryu J, Kim YS (2002) Clinical implications of extendedspectrum beta-lactamase-producing Klebsiella pneumoniae bacteraemia. $J$ Hosp Infect 52: $99-106$

32. Kang CI, Kim SH, Kim DM, Park WB, Lee KD, et al. (2004) Risk factors for and clinical outcomes of bloodstream infections caused by extended-spectrum betalactamase-producing Klebsiella pneumoniae. Infect Control Hosp Epidemiol 25: 860-867.

33. Endimiani A, Luzzaro F, Perilli M, Lombardi G, Colì A, et al. (2004) Bacteremia due to Klebsiella pneumoniae isolates producing the TEM-52 extended-spectrum betalactamase: treatment outcome of patients receiving imipenem or ciprofloxacin. Clin Infect Dis 38: 243-251. [Crossref]

34. Ho PL, Chan WM, Tsang KW, Wong SS, Young K (2002) Bacteremia caused by Escherichia coli producing extended-spectrum beta-lactamase: a case-control study of risk factors and outcomes. Scand J Infect Dis 34: 567-573.

Copyright: (C)2017 Chavada R. This is an open-access article distributed under the terms of the Creative Commons Attribution License, which permits unrestricted use, distribution, and reproduction in any medium, provided the original author and source are credited. 